\title{
Autistic Traits, Empathizing-Systemizing, and Gender Diversity
}

\author{
Olivia Hendriks, $\mathrm{MSc}^{1}$, Yimeng Wei, MSc${ }^{1}$, Varun Warrier ${ }^{2}$, Gareth Richards, $\mathrm{PhD}^{1,2^{*}}$ \\ ${ }^{1}$ School of Psychology, Faculty of Medical Sciences, Newcastle University, UK \\ ${ }^{2}$ Autism Research Centre, Department of Psychiatry, University of Cambridge, UK
}

Conflict of interest: The authors declare that they have no conflict of interest.

* Corresponding author address: School of Psychology, Newcastle University, 4.32 Dame Margaret Barbour Building, Wallace Street, Newcastle upon Tyne, NE2 4DR, UK; email: gareth.richards@ncl.ac.uk

This preprint has not undergone any post-submission improvements or corrections. The Version of Record of this article is published in Archives of Sexual Behavior, and is available online at https://doi.org/10.1007/s10508-021-02251-x 


\section{ABSTRACT}

Previous research indicates a link between autism and transgender and gender-diverse identities, though the association is not yet fully understood. The current study examined autistic traits (Autism Spectrum Quotient [AQ]), empathizing (Empathizing Quotient-Short [EQ-S]), and systemizing (Systemizing Quotient-Short [SQ-S]) in a sample of 89 adults and aimed to test whether gender diverse individuals exhibit cognitive profiles consistent with predictions derived from the Extreme Male Brain theory. As most research has considered only cisgender people, we recruited a more diverse sample by contacting $>200$ UK LGBT + organizations and posting on social media. A range of non-cisgender identities (e.g., transgender male, transgender female, non-binary, genderqueer, transmasculine) and nonheterosexual orientations (e.g., homosexual, bisexual) were represented, and participants were categorized into one of four groups: (1) assigned female at birth but does not identify as female (transgender AFAB) $(n=32),(2)$ cisgender female $(n=21),(3)$ assigned male at birth but does not identify as male (transgender AMAB) $(n=18)$, and $(4)$ cisgender male $(n=18)$. After controlling for age and autism diagnostic status, transgender AFAB participants had marginally higher AQ scores, and significantly higher SQ-S and systemizing-relative-toempathizing (D) scores, compared with the cisgender female group. No such differences were detected between the transgender AMAB and cisgender male groups. Our findings are broadly in line with predictions derived from the Extreme Male Brain theory, though as no transgender AFAB participants reported being heterosexual, it was not possible to determine whether these effects relate specifically to gender identity, to sexual orientation, or to both. Keywords: Autism; Autistic traits; Empathizing-Systemizing; Extreme Male Brain theory; Gender diversity; Transgender 


\section{INTRODUCTION}

Gender identity is distinct from the sex assigned at birth (typically male or female) and represents a person's sense of their own gender. A person who is cisgender has a gender identity that is the same as the sex assigned at birth. There is a range of gender identities, including transgender, non-binary, gender fluid, bigender (and many others). In the current paper, we follow the definition of Warrier et al. (2020, p. 1) and refer to these and other diverse gender identities as "transgender and gender-diverse," i.e. "individuals whose gender does not always correspond to the sex they were assigned at birth." Gender dysphoria, on the other hand, is a strong and persistent dissonance between the sex assigned at birth and one's gender identity (American Psychiatric Association, 2013). Recent evidence suggests although gender dysphoria is quite rare, people who self-identify as transgender or gender diverse represent a large and increasing percentage of the general population (Zhang et al., 2020). Furthermore, whereas gender dysphoria was previously found to affect relatively more birth assigned males than birth assigned females (Zucker \& Lawrence, 2009), this trend appears to have reversed in recent adolescent cohorts, with relatively more birth assigned females than birth assigned males now presenting at gender clinics (Aitken et al., 2015; Zucker, 2017).

An emerging literature has examined the co-occurrence between autism/autistic traits and gender diversity and gender dysphoria (for recent reviews, see Glidden et al., 2016; Øien et al., 2018; Sala et al., 2020; van der Miesen et al., 2016). Autism spectrum conditions are characterized by unusually routine behavior, circumscribed interests, sensory hypersensitivity, difficulties adjusting to unexpected change, and social and communication problems (American Psychiatric Association, 2013), whereas autistic traits are those traits that are related to autism and found approximately normally distributed throughout the general population (Baron-Cohen et al., 2001; Ruzich et al., 2015). Autism spectrum conditions and autistic traits exhibit marked sex differences, with approximately three to four 
males diagnosed as autistic for every one female (Baio et al., 2018; Fombonne, 2009; Hull et al., 2020; Loomes et al., 2017). Research generally points to there being an overrepresentation of autism diagnoses (de Vries et al., 2010; Leef et al., 2019) and elevated autistic traits (Skagerberg et al., 2015) in children and adolescents presenting at gender clinics, and an elevated likelihood of diagnosed gender dysphoria (Hisle-Gorman et al., 2019) and parent-reported gender variant identity (Strang et al., 2014) in autistic children and adolescents. Autistic adolescents have also been shown to report a higher likelihood of a desire to be a different gender to that assigned at birth compared with general population adolescents (van der Miesen et al., 2018).

Until recently, the literature at the intersection between gender diversity and autism in adults was fairly limited (Glidden et al., 2016). However, Warrier et al. (2020) reported an analysis of five independently recruited cross-sectional datasets $(\mathrm{N}=641,860)$ that showed higher rates of autism diagnosis in transgender and gender diverse adults compared to cisgender controls. These findings are consistent with observations of an increased prevalence of autism diagnosis (Heylens et al., 2018) and elevated levels of autistic traits in adults attending gender clinics (Heylens et al., 2018; Jones et al., 2012; Pasterski et al., 2014), although it should be noted that not all studies have observed such effects (e.g., Vermaat et al., 2018; see also Nobili et al., 2018). Warrier et al. (2020) also showed that transgender and gender diverse adults scored higher on a self-report measure of autistic traits, and other researchers have reported elevated levels of autistic traits in adults with self-reported gender diversity (Kristensen \& Broome, 2015; Kung, 2020). Other studies have indicated that autistic adults report elevated rates of gender variance (Cooper et al., 2018; George \& Stokes, 2018) as well as an elevated likelihood of desiring to be a different gender to that assigned at birth (van der Miesen et al., 2018). 
The association between autism (and autistic traits) and gender diversity is gaining clinical and research interest (Glidden et al., 2016; Sala et al., 2020; Strang et al., 2018a, 2020; van der Miesen et al., 2016), and attention to the specific needs of those who are both autistic and have transgender and gender diverse identities (and/or gender dysphoria) appear to be warranted. Although many transgender and gender diverse people do not need nor desire therapeutic support, it is worth noting that individuals who are either autistic (Griffiths et al., 2019; Hollocks et al., 2019) or gender diverse (Millet et al., 2017; Valentine \& Shipherd, 2018) are at increased risk of a range of negative outcomes such as stress, isolation, poor self-esteem, anxiety, depression, alcohol/drug addiction, and suicidality. Additionally, the status of being a minority within a minority that is true of autistic people who are transgender or gender diverse is likely to result in even greater challenges (Coleman-Smith et al., 2020).

It has been suggested that the presentation of autism in many cisgender girls and women differs from that of cisgender boys and men (Bargiela et al., 2016; Dworzynski et al., 2012; Lai et al., 2017; see also Strang et al., 2020), and that people's gender diversity is sometimes questioned because of their autism diagnosis (Coleman-Smith et al., 2020; Strang et al., 2018b). Efficacy of care for autistic individuals who choose to seek treatment for gender dysphoria may additionally be affected by some of the core features of autism. For instance, it could be that autistic individuals attending gender clinics experience increased difficulty in communicating their current needs to medical practitioners (Strang et al., 2018a). Such ideas suggest that a better understanding of the association between autism and gender diversity is necessary to provide more effective support when it is required.

An idea of potential relevance to explaining the connection between transgender and gender diverse identities and the autism spectrum is that of Empathizing-Systemizing (E-S) theory (see Baron-Cohen, 2003). It posits that two constructs (empathizing and systemizing) 
underly human cognitive sex differences, and the Extreme Male Brain (EMB) theory (BaronCohen, 2002; Greenberg et al., 2018) extends this to the idea that autism is characterized by an exaggerated form of the male-typical cognitive profile. Empathizing is the ability to identify and respond appropriately to emotions and thoughts in others, whereas systemizing is the drive to analyze and build systems based on input-operation-output rules. Evidence for the veracity of these theories is provided by observations that, on average, non-autistic males score higher than non-autistic females on systemizing $(d=.31)$, that non-autistic females score higher than non-autistic males on empathizing $(d=.39)$, and that autistic males and females score higher in systemizing (males: $d=.30$; females: $d=.39$ ) and lower in empathizing (males: $d=.41$; females: $d=.51$ ) than non-autistic sex-matched controls (Greenberg et al., 2018). Sex differences observed for these measures also appear attenuated in autistic adults compared to non-autistic adults (Baron-Cohen et al., 2014; Greenberg et al., 2018). The difference in standardized empathizing and systemizing (D-score) is higher in non-autistic males than non-autistic females $(d=.94)$, and higher in autistic males and females than non-autistic males and females, respectively (males: $d=1.34$; females: $d=1.48$ ) (Baron-Cohen et al., 2014 [present authors' calculations]). Interestingly, D-scores have been reported to explain 19 times more variance in autistic traits (43\%) than other demographic variables, including sex (Greenberg et al., 2018), yet have typically not been examined in studies that investigate associations between autism and gender diversity.

Jones et al. (2012) were the first to suggest that predictions about the association between autism and transgender identity could be derived from the EMB theory. Elevated exposure to fetal androgens is posited to increase the likelihood of autism (Baron-Cohen et al., 2004, 2015) as well as a male gender identity (Hines, 2004); thus it can be predicted that autism and transgender identity in birth assigned females will occur at a rate greater than chance (see also van der Miesen et al., 2016). In line with this prediction, Jones et al. (2012) 
reported that transgender males had higher levels of autistic traits than cisgender females and that transgender females and cisgender males did not differ in this regard. Di Ceglie et al. (2014) then reported that empathizing in adolescent transgender males and transgender females was lower than that of cisgender females, though they did not find any differences for systemizing. Although findings in this area have been equivocal (for a review, see van der Miesen et al., 2016), Stagg and Vincent (2019) and Kung (2020) recently confirmed the pattern of findings that would be predicted by the EMB theory: in both studies, higher empathizing and lower systemizing were found in transgender males relative to cisgender females, but no significant differences were found between transgender females and cisgender males. Although Warrier et al. (2020) reported lower empathizing and higher systemizing in transgender and gender diverse individuals compared with cisgender individuals in a very large sample, no analysis by birth assigned sex was possible in that study.

So far, relatively few studies have examined gender identities other than those that fit a binary classification (i.e., transgender males, transgender females, cisgender males, and cisgender females) in relation to autism/autistic traits. Kristensen and Broome (2015) examined a range of self-reported gender identities (e.g., androgyne, trans, transsexual, third gender), and reported that autistic traits were particularly elevated in individuals identifying as gender-queer (i.e., a person who does not subscribe to the conventional gender distinctions of male or female, but identifies as neither, both, or a combination of male and female genders). Furthermore, autistic individuals are more likely than neurotypical individuals to report their gender identity as atypical (i.e., not congruent with their birth sex) (Bejerot \& Eriksson, 2014; George \& Stokes, 2018). Self-identified non-binary individuals, on average, also score higher on self-report measures of autistic traits and systemizing, and lower on selfreport measures of empathizing (Kung, 2020; Stagg \& Vincent, 2019). 
The present study aims to address gaps in the literature regarding how traits related to autism are associated with gender diversity by sampling a broad range of gender identities. Instead of comparing only transgender males and transgender females (or non-binary) participants with cisgender males and cisgender females, we used an online survey, and hypotheses derived from the EMB theory, to investigate the relationship between various transgender and gender diverse identities and autistic traits, empathizing, and systemizing. Fundamentally, the E-S and EMB theories are about cognition along two axes (empathizing and systemizing) that impact behavior. The underlying mechanisms that shape this cognition are diverse and include genetics, prenatal hormones, and postnatal experience. Notably, many of these factors also underlie those associated with autism, a condition characterized by differences in cognition. Thus, the E-S theory and, its extension, the EMB theory suggest that the mechanisms underlying normative sex differences (i.e., those factors which give rise to sex differences in empathizing and systemizing) contribute, at least in part, to likelihood for autism, with empirical support emerging from self- and parent-report measures of these two axes. Both sex differences and rates of autism are different in transgender and gender diverse individuals. However, we again emphasize that the mechanisms underlying gender identity are equally complex. As such, one prediction emerging from the E-S and EMB theories is that transgender and gender diverse individuals will score differently on these two axes of cognition compared to cisgender individuals. Considering this alongside the more specific hypotheses derived from EMB theory by Jones et al. (2012) and Nobili et al. (2018), we predicted that, compared to cisgender females, transgender AFAB participants would have more male-typical scores on traits associated with autism. More precisely, we predicted that transgender AFAB participants would have higher scores for autistic traits (AQ) and systemizing (SQ-S), lower scores for empathizing (EQ-S), and a higher systemizing-relativeto-empathizing (D) score, and that there would be no significant differences on these 
variables between transgender AMAB individuals and cisgender males. Given that the E-S and EMB theories make no predictions on sex vis-à-vis gender, we hesitate to speculate regarding possible differences between groups of gender diverse individuals and hence do not test if transgender AFAB and transgender AMAB individuals score differently to each other.

\section{METHOD}

\section{Participants}

To ensure that a broad range of gender identities were represented, we contacted $>200$ lesbian, gay, bisexual, transgender, queer+ $\left(\mathrm{LGBTQ}^{+}\right)$support groups across the UK via email to ask for assistance in advertising our survey via their social media channels. The email explained that the study would take approximately 20 minutes, that it would examine gender identity, personality, and prenatal testosterone exposure, and that it was being conducted as part of an M.Sc. dissertation project at Newcastle University. We further clarified that the study had been granted approval by Newcastle University, and provided the following as a suggested message to share on social media: "Interested in gender and personality? Please consider taking part in a 20-minute online survey for a chance to win a $£ 25.00$ Amazon voucher. Please follow this link if you would like to take part:” (followed by the survey link). To ensure we enlisted similar numbers of cisgender and gender diverse participants, and that each of the four groups (cisgender females, cisgender males, transgender $\mathrm{AFAB}$, transgender $\mathrm{AMAB}$ ) examined was represented by a broad range of sexual orientations, we also recruited participants via online survey exchanges (e.g., SurveyCircle, PollPool). No exclusion criteria were specified other than that participants should be at least 18 years of age.

A total of 133 people accessed the survey, 101 of whom completed the AQ. Of these, 9 did not report either their assigned and/or current gender (e.g., responded with "other" or "prefer not to say,") and 3 reported diagnosed/suspected gender dysphoria/gender identity 
disorder as well as the same current gender identity as that assigned at birth. These participants were therefore removed from further analysis, leaving a sample of 89 . The remaining participants were categorized into one of four groups: (1) assigned female at birth but does not identify as female (transgender AFAB) $(n=32),(2)$ cisgender female $(n=21)$, (3) assigned male at birth but does not identify as male (transgender AMAB) ( $\mathrm{n}=18)$, or (4) cisgender male $(\mathrm{n}=18)$.

\section{Measures}

We used the Autism-Spectrum Quotient (AQ; Baron-Cohen et al. 2001) to quantify autistic traits. The AQ is a 50-item self-report measure of autistic traits that has good testretest reliability and can differentiate between autistic adults and non-autistic adults (BaronCohen et al., 2001, 2014; Woodbury-Smith et al., 2005). The measure includes four response options (“definitely agree”, "slightly agree”, "slightly disagree”, and “definitely disagree”); 26 of the items are reverse scored, and one point is given for each response either slightly or strongly endorsing an autistic trait. Scores range from 0 to 50 , with higher scores indicating presence of more traits that are characteristic of autism. Example items include "I prefer to do things the same way over and over again", and "In a social group, I can easily keep track of several different people's conversations" (the latter being reverse scored). Internal consistency for the total score in the current study was high (Cronbach's $\alpha=0.90)$.

The short forms of the Empathy Quotient (EQ-Short [EQ-S]) and Systemizing Quotient (SQ-Short [SQ-S]) were used to measure empathizing and systemizing, respectively (Wakabayashi et al., 2006). The EQ-S is a 22-item version of the original 60-item EQ (Baron-Cohen \& Wheelwright 2004), and the SQ-S is a 25-item version of the original 60item SQ (Baron-Cohen et al., 2003). The short forms were used as they are strongly correlated with the full-length scales (Wakabayashi et al., 2006), and because they are quicker to administer. There are four response options for both measures: "strongly agree", 
"slightly agree", "slightly disagree", and "strongly" disagree". For the EQ-S, one point is given for a response that slightly endorses an empathizing trait whereas two points are given for a response that strongly endorses an empathizing trait; for the SQ-S, one point is given for a response that slightly endorses a systemizing trait, and two points are given for a response that strongly endorses a systemizing trait. Example items include "I am good at predicting how someone will feel" (EQ-S), and "I am fascinated by how machines work" (SQ-S). Six items from the EQ-S and 13 items from the SQ-S are reverse-scored. Scores for the EQ-S range from 0 to 44 (higher scores indicating higher empathizing), whereas scores for the SQS range from 0 to 50 (higher scores indicating higher systemizing). Internal consistency for the total scores in the current study was high (EQ-S, $\alpha=0.91$; SQ-S, $\alpha=0.86$ ). The standardized mean difference (D-score) between empathizing and systemizing was calculated from standardized EQ-S (E) and SQ-S (S) scores as D-score $=(S-E) / 2$.

\section{Procedure}

Participants were informed that the purpose of the study was to assess whether gender identity is related to personality and prenatal testosterone exposure, and that it may help improve our understanding of gender identity as well as individual difference characteristics with which it may be related. We explained that some questions (e.g., gender assigned at birth, current gender identity, ethnicity, sexual orientation, diagnoses of clinical conditions) relate to potentially sensitive topics, and that they would be free to withdraw from the study at any point without specifying a reason and without penalty. Participants were provided with contact details for the research team, though we additionally stated that we would not be able to inform individuals of their specific questionnaire scores. We also explained that data would be anonymous, that the dataset could be made available online alongside an academic publication, and that participants would be asked to give informed consent if deciding to take part in the study. 
After providing informed consent, participants were asked to report their age (18-99 years), gender assigned at birth ("male," "female," "other [please specify],") current gender identity ("male," "female," "other [please specify],") sexual orientation ("heterosexual," "homosexual," "bisexual," "other [please specify],") and ethnicity (“Asian/Asian British,” "Black/Black British," "Black other," "Chinese, Middle/Near Eastern," "Mixed ethnicity," "White.") After this they were administered the AQ, EQ-S, and SQ-S, before reporting whether they had been diagnosed with autism and/or gender dysphoria/gender identity disorder, and whether they suspected they were autistic and/or had gender dysphoria/gender identity disorder. The order of questions was arranged to avoid priming effects, and the information sheet made no explicit reference to autism. Finally, participants were asked to measure the lengths of their second and fourth fingers for a separate study of gender diversity and digit ratio (2D:4D) (see Richards et al. 2020).

The online survey (hosted by Qualtrics) took approximately 20 minutes to complete. Participants were thanked and debriefed on completion, and signposting information was provided for sources of support for autism and gender identity concerns. Ethical approval was granted by the Faculty of Medical Sciences Research Ethics Committee, Newcastle University (approval number: 1689/12185/2019), and the research was carried out in accordance with the Declaration of Helsinki as revised in 2000.

\section{Statistical Analysis}

The predictor variables were gender assigned at birth (male or female) and current gender (same as assigned at birth or different to assigned at birth), and the outcomes were autistic traits (AQ total score), empathizing (EQ-S total score), systemizing (SQ-S total score), and systemizing-relative-to-empathizing (D-score). AQ, EQ-S, and SQ-S total scores were computed by summing all items from each of the respective scales. We used chi-square tests to ascertain whether autism status (diagnosed/suspected or not diagnosed/suspected) and 
ethnicity (White or non-White) differed across the four gender groups (transgender AFAB, cisgender female, transgender $\mathrm{AMAB}$, and cisgender male), and whether diagnosed/suspected autism was associated with diagnosed/suspected gender dysphoria/gender identity disorder; the effect size was reported as Cramér's V and interpreted according to the criteria specified by Cohen (1988) (i.e., . $10=$ small; $.30=$ medium; $.50=$ large). We then used one-way analysis of variance (ANOVA) followed by least significant difference tests to determine whether the four gender groups differed significantly in age. We also conducted Pearson's tests to examine the pattern of intercorrelations between AQ, EQ-S, SQ-S, and D.

As the predictor variables (sex assigned at birth: male or female; current gender: same or different) were categorical and independent, and the outcome variables (AQ, EQ-S, SQ-S, and D) were continuous and approximately normally distributed, we proceeded with a between participants multivariate analysis of covariance (MANCOVA). More specifically, we conducted a MANCOVA followed by individual ANCOVAs for AQ, EQ-S, and SQ-S, but used only ANCOVA when assessing D-scores, as D is derived from two of the other outcome variables (i.e., EQ-S and SQ-S). Age (continuous variable) and autism diagnostic status (diagnosed/suspected autism or not diagnosed/suspected autism) were included as covariates. We included age as a covariate because the age at which a transgender male identity is first reported is typically lower than the age at which a transgender female identity is first reported (Nieder et al., 2011); we included autism status as a covariate so that we could establish whether any observed effects relating to AQ, EQ-S, SQ-S, or D-score were independent of group differences in autism prevalence. Simple effects tests (with Bonferroni adjustment for alpha inflation) were then used to examine for differences between the transgender AFAB group and cisgender female group and between the transgender AMAB group and the cisgender male group. Effect sizes were reported as $\eta_{\mathrm{p}}^{2}(.01=$ small; $.06=$ 
medium; .14 = large; Cohen 1988). Data were analyzed using IBM SPSS version 27, and results were considered statistically significant at $p<.05$.

\section{RESULTS}

Twenty-two participants in the transgender AFAB group further specified their current gender identity; the following frequencies were observed: agender $(n=2)$, genderqueer $(n=3)$, genderqueer/fluid $(n=1)$, non-binary $(n=11)$, non-binary or demi-girl $(n=1)$, non-binary gender fluid $(n=2)$, transmasculine $(n=1)$, transmasculine non-binary $(n$ $=1$ ). In the transgender AMAB group, only three participants further specified their current gender identity ( $\mathrm{n}=1$ gender fluid; $\mathrm{n}=2$ non-binary). Most participants were White, and a wide range of ages and sexual orientations were represented; most gender diverse participants reported that they found the survey via links from LGBTQ+ support groups, whereas most cisgender participants did not indicate this (see Table 1 for descriptive statistics).

\section{Associations Between Gender Diversity, Autistic Traits, and Empathizing-Systemizing}

Means and SD for AQ, EQ-S, SQ-S, and D-scores stratified by gender group are shown in Table 2. A chi-square test revealed the prevalence of diagnosed/suspected autism differed significantly across the four gender groups, $\chi^{2}(3, \mathrm{~N}=89)=8.72, p=.03, \mathrm{~V}=.31$. Examination of the observed frequencies compared to those expected by chance alone indicated that there was a relatively high prevalence of autism within the transgender AFAB group and a relatively low prevalence of autism in the cisgender male group. Participants' ethnicity (White or non-White) did not differ across the four groups, $\chi^{2}(3, \mathrm{~N}=89)=1.65, p=$ $.65, \mathrm{~V}=.14$. One-way ANOVA determined that there was a significant difference in participants' age across the four groups, $F(3,85)=4.32, p=.01, \eta_{\mathrm{p}}^{2}=.13$. Post hoc least significant difference tests indicated that transgender AFAB participants were younger than transgender AMAB participants $(p<.01)$ and cisgender males $(p=.01)$, and that cisgender females were younger than transgender AMAB participants $(p=.03$ ) (all other comparisons 
were not statistically significant). Interestingly, diagnosed/suspected autism was not significantly associated with diagnosed/suspected gender dysphoria/gender identity disorder, $\chi^{2}(1, \mathrm{~N}=89)=1.90, p=.17, \mathrm{~V}=.15$.

When examined in the whole sample, the autism-related questionnaire measures showed a pattern of high intercorrelations. AQ was negatively correlated with EQ-S, $r(83)=$ $.78, p<.001$, and positively correlated with SQ-S, $r(81)=.43, p<.001$, and D-score, $r(81)=$ $.79, p<.001$. EQ-S was weakly negatively correlated with SQ-S, $r(81)=-.22, p=.04$, and Dscores were positively correlated with SQ-S, $r(81)=.73, p<.001$, and negatively correlated with EQ-S, $r(81)=-.83, p<.001$. A similar pattern was observed for each of the four gender identity groups when examined individually (statistical output is not reported here in the interests of brevity).

We used a 2 (gender assigned at birth: male or female) $\times 2$ (current gender: same as assigned or different to assigned) between participants MANCOVA with age and autism status (diagnosed/suspected autism or no diagnosed/suspected autism) as covariates, and AQ, EQ-S, and SQ-S as outcome variables. Box's $M$ test was non-significant, indicating homogeneity of variance-covariance matrices, $M=14.15, p=.79$, and Levene's test was nonsignificant for each outcome variable (AQ: $F[3,79]=1.44, p=.24$; EQ-S: $F[3,79], p=.88$; SQ-S: $F[3,79]=.79, p=.50)$, indicating homogeneity of variances across groups. Although diagnosed/suspected autism was a significant predictor of variance in the outcome variables, Wilks' $\lambda=.58, F(3,75)=18.19, p<.001, \eta_{\mathrm{p}}^{2}=.42$, none of the other predictors or covariates were significant: gender assigned at birth, Wilks' $\lambda=.97, F(3,75)=.77, p=.52$, $\eta_{\mathrm{p}}{ }^{2}=.03$; current gender, Wilks' $\lambda=.92, F(3,75)=2.06, p=.11, \eta_{\mathrm{p}}{ }^{2}=.08$; gender assigned at birth $\times$ current gender: Wilks' $\lambda=.94, F(3,75)=1.57, p=.20, \eta_{\mathrm{p}}{ }^{2}=.06$; age: Wilks' $\lambda=$ $.95, F(3,75)=1.46, p=.23, \eta_{\mathrm{p}}{ }^{2}=.06$. 
We elected to follow the MANCOVA with separate ANCOVAs (Table 3). Our reasoning for this was two-fold: first, given the relatively small sample, we wanted to avoid excluding participants for whom complete data were not available for all outcome variables, and (2) because we had a priori predictions regarding which groups would differ (and in which direction) for the individual outcome variables. Each ANCOVA model met the assumptions of homogeneity of variance and homogeneity of regression slopes.

Diagnosed/suspected autism was a significant predictor of high AQ and low EQ-S scores, whereas having a gender identity different from the sex assigned at birth predicted high SQ-S scores. When we computed a similar ANCOVA model with D-score as the outcome variable, the assigned gender $\times$ current gender interaction term was significant (see Fig. 1).

We used simple effects tests with Bonferroni correction (required $\alpha: p<.025$ ) to determine whether the adjusted means for each outcome variable differed between the transgender $\mathrm{AFAB}$ group and the cisgender female group, and whether they differed between the transgender $\mathrm{AMAB}$ group and the cisgender male group. Although the transgender $\mathrm{AFAB}$ and cisgender female groups did not differ for EQ-S $\left(F[1,79]=.88, p=.35, \eta_{\mathrm{p}}{ }^{2}=.01\right)$, higher scores were observed in the transgender AFAB group for AQ $(F[1,83]=4.61, p=$ $\left..04, \eta_{\mathrm{p}}^{2}=.05\right), \mathrm{SQ}-\mathrm{S}\left(F[1,77]=9.84, p<.01, \eta_{\mathrm{p}}^{2}=.11\right)$, and $\mathrm{D}\left(F[1,77]=6.42, p=.01, \eta_{\mathrm{p}}^{2}\right.$ $=.08$ ) (though note that the effect for AQ did not survive Bonferroni correction). There were no significant differences between the transgender AMAB group and the cisgender male group for any of the outcomes examined: AQ, $F(1,83)=.03, p=.87, \eta_{\mathrm{p}}^{2}<.001$; EQ-S, $F(1$, $79)=1.32, p=.26, \eta_{\mathrm{p}}^{2}=.02 ; \mathrm{SQ}-\mathrm{S}, F(1,77)=.19, p=.67, \eta_{\mathrm{p}}^{2}<.01 ; \mathrm{D}, F(1,77)=.44, p=$ $.51, \eta_{\mathrm{p}}^{2}=.01$

\section{DISCUSSION}

The current study examined autistic traits, empathizing, and systemizing in an adult sample representing a broad range of gender identities and sexual orientations. In line with 
our predictions, there were no significant differences between the transgender AMAB and cisgender male groups but AQ, SQ-S, and D-scores were higher, and EQ-S scores were lower, in transgender AFAB participants than cisgender female participants. Although the effect for EQ-S was not significant, and that relating to AQ did not survive Bonferroni correction, the overall pattern is consistent with predictions derived from the EMB theory (see Jones et al., 2012). It should be noted, however, that the transgender AFAB group was the only one for which the mean AQ score $(M=25.88, S D=10.25)$ approached the suggested cut-off points of 26 (Woodbury-Smith et al., 2005) or 32 (Baron-Cohen et al., 2001) that may indicate clinical concern, and that this group also included the highest prevalence of diagnosed and/or suspected autism (43.8\%). However, as less than half (6 of 14) of those who indicated diagnosed and/or suspected autism reported that they had received a diagnosis, this pattern of results remains consistent with the notion that there is an elevated prevalence of undiagnosed autism associated with transgender AFAB status.

Although the current findings align with those of research linking gender diversity with autism/autistic traits, potential explanations for this co-occurrence remain speculative (for an overview, see van der Miesen et al. 2016). The EMB theory, which suggests that autism represents a hyper-masculinized cognitive profile (i.e., systemizing $>>$ empathizing) (Baron-Cohen, 2002, 2003; Baron-Cohen et al., 2005; Greenberg et al., 2018), implies that gender diversity in autistic birth assigned females could be an expression of an "extreme male" characteristic. Consistent with this theory is the idea that autism and gender diversity in birth assigned females share a biological underpinning in terms of atypical fetal sex hormone exposure. Evidence for this comes from studies showing that second trimester amniotic testosterone levels correlate positively with autistic traits (Auyeung et al., 2009, 2010, 2012; though see also Kung et al., 2016, who did not replicate this effect) and systemizing (Auyeung et al., 2006) and negatively with empathizing (Chapman et al., 2006), 
and that elevated levels of androgens (Baron-Cohen et al., 2015) and estrogens (Baron-Cohen et al., 2019) are present in the amniotic fluid of males who develop autism.

The finding of the current study that elevated autistic traits, systemizing, and systemizing-relative-to-empathizing were present in the transgender AFAB group but not in the transgender $\mathrm{AMAB}$ group is consistent with predictions of the EMB theory, as are findings of studies that report a stronger association between autism and gender diversity in birth assigned females than birth assigned males (Cooper et al., 2018; Dewinter et al., 2017; Jones et al., 2012; Kung, 2020; van der Miesen et al., 2018 [adolescent sample]; see also Nobili et al., 2018; Vermaat et al., 2018). However, other studies have reported no significant sex difference (e.g., Hisle-Gorman et al., 2019; Pasterski et al., 2014; Skagerberg et al., 2015; Strang et al., 2014; van der Miesen et al., 2018 [adult sample]) or even a higher prevalence of autism in birth assigned males presenting at gender clinics (de Vries et al., 2010; Heylens et al., 2018). Considering the equivocal pattern of results in this area, future studies utilizing large sample sizes should examine differences in prevalence of autism diagnosis, autistic traits, and empathizing and systemizing across transgender AFAB, transgender $\mathrm{AMAB}$, cisgender female, and cisgender male groups. One such possibility would be to examine data from the BBC Internet Study (see Reimers, 2007), as participants completed self-report measures of empathizing and systemizing (Manning, Baron-Cohen, et al., 2010a, 2010b) and also reported their birth assigned sex as well as gender identity (Manning et al., 2020). Additionally, it could be useful to examine the question meta-analytically (Warrier et al., 2020).

It should be noted that the EMB theory does not explain why autistic males report higher rates of gender dysphoria relative to typically developing males (van der Miesen et al., 2016), and a recent trend for birth assigned females to be more likely than birth assigned males to present at gender clinics (Aitken et al., 2015) may suggest a broader societal 
phenomenon rather than one that can be explained in terms of differences in prenatal testosterone levels (van der Miesen et al., 2018). Furthermore, there may be reasons other than those biological for explaining sex differences in autism diagnosis: for instance, it has been noted that diagnostic algorithms were developed based on predominantly male samples, and so may not be sensitive to detecting autism in females and gender diverse individuals (Ratto, 2021), which can lead to a diagnostic bias in favor of males (Loomes et al., 2017). It is also acknowledged that some autistic adults with transgender or gender diverse identities report finding terminology related to the EMB theory unhelpful, and suggest that the notion of being categorized as having an extreme male brain could increase their sense of dysphoria (Coleman-Smith et al., 2020). Future work should investigate this consideration and aim to determine improved ways of supporting this population.

Although biological factors are clearly important in the development of one's gender identity (Hines, 2004; Meyer-Bahlburg, 2005), different expectations of males and females as regards adherence to socially-derived gender roles should not be overlooked (George \& Stokes, 2018; Kanfiszer et al., 2017). Notably, autistic females report higher masculinity, lower femininity, and lower gender identification than autistic males (Cooper et al., 2018), often prefer socializing with males than females (Bargiela et al., 2016), report elevated rates of tomboyism in childhood and adolescence (Bejerot \& Eriksson, 2014; Ingudomnukul et al., 2007), and may not automatically identify with the construct of femininity as strongly as nonautistic females do (Kanfiszer et al., 2017). However, it remains unclear whether such factors are causes and/or consequences (or merely correlates) of gender diversity, and indeed autistic features (e.g., intense/obsessional interests; Landén \& Rasmussen, 1997; Zucker et al., 2017) could be mistaken for gender dysphoria symptoms and vice versa (Heylens et al., 2018). Additionally, other social processes (e.g., social and communication difficulties associated with autism; Landén \& Rasmussen [1997]) may play important roles in explaining the link 
between autism and gender dysphoria. This suggestion is supported by George and Stokes' (2018) finding that gender dysphoria traits were most strongly associated with the Social Skill $(r=.35)$ and Communication $(r=.36)$ subscales of the AQ (although a comparably sized correlation was also observed for cognitive inflexibility $[r=.34)$. However, it should be noted that self-report measures of autistic traits have not been validated in gender diverse populations, that they may lack specificity, and that elevated scores on such measures could reflect social difficulties (e.g., challenges in relating to peers) associated with gender dysphoria rather than autism (Heylens et al., 2018; Skagerberg et al., 2015).

A strength of the current research is that we sampled a wide range of gender identities and sexual orientations (Ratto, 2021), although it should be acknowledged that individuals who are members of support groups are unlikely to be truly representative of autistic and/or transgender and gender diverse people. This consideration might be reflected in the observation that not only were the rates of diagnosed/suspected autism higher than the general adult population average (approximately 1-2\%; Brugha et al., 2016) in the transgender AFAB (43.8\%) and transgender AMAB (22.2\%) groups, but they were also considerably higher than might be expected in the cisgender female (28.6\%) and cisgender male $(5.6 \%)$ groups. It is unclear why this is the case, as we deliberately did not approach groups that related specifically to autism and avoided referring to autism when advertising the study on social media. However, as a considerable proportion of our participants found the study via links from LGBT+ support groups, it is possible that this higher prevalence of autism within our sample relies, at least in part, on the observation that there is greater variability in the sexual orientation of autistic adults compared to non-autistic adults (Bejerot \& Eriksson, 2014; Cooper et al., 2018; Dewinter et al., 2017). Unfortunately, the terminology we used regarding sexual orientation (heterosexual, homosexual, etc.) can create confusion in the current context, particularly as we did not specify whether this should relate to one's sex 
assigned at birth or one's current gender identity; it would therefore have been more informative to use less ambiguous terms such as "gynophilic" and "androphilic." Furthermore, as no participants in the transgender AFAB group in our study reported that they were heterosexual, it was not possible to reliably determine whether the effects observed here relate to gender identity, sexual orientation, or both. This is an important limitation considering that Vermaat et al. (2018) found that AFAB participants with non-heterosexual orientations in their sample reported the highest levels of autistic traits, and also because George and Stokes (2018) observed that gender dysphoria traits were a significant mediator of the association between AQ score and sexual orientation. Furthermore, it is currently unknown whether autistic traits are qualitatively different in gender diverse individuals compared to cisgender individuals, and this needs to be investigated. This is particularly important considering that those with social anxiety disorder, on average, obtain higher AQ scores than population controls (Hoekstra et al., 2008), and anxiety disorders are frequently present in both autistic (Hollocks et al., 2019) and gender diverse people (Millet et al., 2017).

A further limitation of the current study is that the relatively small sample size necessitated the use of a binary (cisgender/non-cisgender) approach to data analysis that does not consider the extensive variation that is present. Unfortunately, our method for recruiting participants made it unfeasible to obtain a larger sample. Whereas a similar online study (Kristensen \& Broome, 2015) recruited a larger number of participants (included in main analysis: $\mathrm{n}=446$ ) through snowball sampling, we contacted every LGBTQ+ support group in the UK of which we were aware, and so could not add more participants without contacting organizations from elsewhere (which could increase the heterogeneity of our sample in unpredictable ways). Although this is a limitation, the fact that we still observed statistically significant differences consistent with those already reported in the literature provides evidence that these effects are robust. It should, however, also be acknowledged that we did 
not assess participants' hormonal treatment status. This may be a particularly important oversight because, although Nobili et al. (2020) reported that autistic traits remain stable following cross-sex hormone treatment, they also found that transgender AFAB adults were more likely than transgender AMAB adults to report clinically significant levels of autistic traits post-treatment even though there was no such difference at baseline.

\section{Implications}

Research into this area is important because a greater understanding of the association between autism and transgender and gender diverse identities could improve processes relating to detection, diagnosis, and support. Furthermore, there is much that we do not know about how autistic traits impact the support needs of gender diverse individuals. Lack of understanding of one's own and others' emotions, problems interpreting social cues and inability to effectively communicate one's thoughts are just some of many obstacles that may adversely interfere with therapeutic gender treatments in those with autistic features (Glidden et al., 2016; Jacobs et al., 2014). As has been suggested by others (e.g., George \& Stokes, 2018), it is therefore vital to establish an improved understanding of core autistic traits present in those who are both autistic and gender diverse, as this could help provide each person with the most effective support for their specific needs.

Taken together, the findings of the current study are broadly in line with predictions derived from the EMB theory of autism. However, given the unique challenges faced by those who are both autistic and gender diverse (Strang et al., 2018b) further research in this area is needed regardless of what the biological, cultural, and/or societal antecedents might be.

\section{ACKNOWLEDGEMENTS}

The authors would like to thank each person who participated in this research, as well as those who helped us to advertise the survey. We would also like to thank Dr Darren 
Johnson (School of Psychology, Newcastle University) for helpful advice during study design. The research was completed in part fulfilment of Olivia Hendriks and Yimeng Wei's MSc Foundations in Clinical Psychology dissertation projects.

\section{COMPLIANCE WITH ETHICAL STANDARDS}

The authors declare that they have no conflict of interest. All procedures performed in studies involving human participants were in accordance with the ethical standards of the Faculty of Medical Sciences Research Ethics Committee, Newcastle University (approval number: 1689/12185/2019) and with the 1964 Helsinki declaration and its later amendments or comparable ethical standards. All participants provided informed consent before completing the survey.

\section{AUTHOR CONTRIBUTIONS}

Gareth Richards, Olivia Hendriks, and Yimeng Wei designed the study and collected the data. Gareth Richards analysed the data and wrote the first draft, Varun Warrier revised the manuscript for important intellectual content, and all authors read and approved the manuscript. 


\section{REFERENCES}

Aitken, M., Steensma, T. D., Blanchard, R., VanderLaan, D. P., Wood, H., Fuentes, A., Spegg, C., Wasserman, L., Ames, M., Fitzsimmons, C. L., Leef, J. H., Lishak, V., Reim, E., Takagi, A., Vinik, J., Wreford, J., Cohen-Kettenis, P. T., de Vries, A. L. C., Kreukels, B. P. C., \& Zucker, K. J. (2015). Evidence for an altered sex ratio in clinic-referred adolescents with gender dysphoria. Journal of Sexual Medicine, 12(3), 756-763. https://doi.org/10.1111/jsm.12817

American Psychiatric Association. (2013). Diagnostic and statistical manual of mental disorders: DSM-5. (5th ed.). American Psychiatric Association.

Auyeung, B., Ahluwalia, J., Thomson, L., Taylor, K., Hackett, G., O’Donnell, K. J., \& BaronCohen, S. (2012). Prenatal versus postnatal sex steroid hormone effects on autistic traits in children at 18 to 24 months of age. Molecular Autism, 3(1), 17. https://doi.org/10.1186/2040-2392-3-17

Auyeung, B., Baron-Cohen, S., Ashwin, E., Knickmeyer, R. C., Taylor, K., \& Hackett, G. (2009). Fetal testosterone and autistic traits. British Journal of Psychology, 100(1), 1-22. https://doi.org/10.1348/000712608x311731

Auyeung, B., Baron-Cohen, S., Chapman, E., Knickmeyer, R. C., Taylor, K., \& Hackett, G. (2006). Foetal testosterone and the child systemizing quotient. European Journal of Endocrinology, 155(suppl_1), S123-S130. https://doi.org/10.1530/eje.1.02260

Auyeung, B., Taylor, K., Hackett, G., \& Baron-Cohen, S. (2010). Foetal testosterone and autistic traits in 18 to 24-month-old children. Molecular Autism, 1(1), 11. https://doi.org/10.1186/2040-2392-1-11

Baio, J., Wiggins, L., Christensen, D. L., Maenner, M. J., Daniels, J., Warren, Z., KurziusSpencer, M., Zahorodny, W., Robinson Rosenberg, C., White, T., Durkin, M. S., Imm, P., 
Nikolaou, L., Yeargin-Allsopp, M., Lee, L.-C., Harrington, R., Lopez, M., Fitzgerald, R. T., Hewitt, A., ... Dowling, N. F. (2018). Prevalence of autism spectrum disorder among children aged 8 years - Autism and Developmental Disabilities Monitoring Network, 11 Sites, United States, 2014. Morbidity and Mortality Weekly Report Surveillance Summaries, 67(6), 1-23. https://doi.org/10.15585/mmwr.ss6706a1

Bargiela, S., Steward, R., \& Mandy, W. (2016). The experiences of late-diagnosed women with autism spectrum conditions: An investigation of the female autism phenotype. Journal of Autism and Developmental Disorders, 46(10), 3281-3294. https://doi.org/10.1007/s 10803-016-2872-8

Baron-Cohen, S. (2002). The extreme male brain theory of autism. Trends in Cognitive Sciences, 6(6), 248-254. https://doi.org/10.1016/S1364-6613(02)01904-6

Baron-Cohen, S. (2003). The essential difference: Men, women and the extreme male brain. Allen Lane/Penguin.

Baron-Cohen, S., Auyeung, B., Nørgaard-Pedersen, B., Hougaard, D. M., Abdallah, M. W., Melgaard, L., Cohen, A. S., Chakrabarti, B., Ruta, L., \& Lombardo, M. V. (2015). Elevated fetal steroidogenic activity in autism. Molecular Psychiatry, 20(3), 369-376. https://doi.org/10.1038/mp.2014.48

Baron-Cohen, S., Cassidy, S., Auyeung, B., Allison, C., Achoukhi, M., Robertson, S., Pohl, A., \& Lai, M.-C. (2014). Attenuation of typical sex differences in 800 adults with autism vs. 3,900 controls. PLoS ONE, 9(7). https://doi.org/10.1371/journal.pone.0102251

Baron-Cohen, S., Knickmeyer, R. C., \& Belmonte, M. K. (2005). Sex differences in the brain: Implications for explaining autism. Science, 310(5749), 819-823. https://doi.org/10.1126/science.1115455

Baron-Cohen, S., Lutchmaya, S., \& Knickmeyer, R. C. (2004). Prenatal testosterone in mind. 
MIT Press.

Baron-Cohen, S., Richler, J., Bisarya, D., Gurunathan, N., \& Wheelwright, S. (2003). The systemizing quotient: An investigation of adults with Asperger syndrome or highfunctioning autism, and normal sex differences. Philosophical Transactions of the Royal Society Biological Bciences, $\quad$ B58(1430), $\quad$ 361-374. https://doi.org/10.1098/rstb.2002.1206

Baron-Cohen, S., Tsompanidis, A., Auyeung, B., Nørgaard-Pedersen, B., Hougaard, D. M., Abdallah, M. W., Cohen, A., \& Pohl, A. (2020). Foetal oestrogens and autism. Molecular Psychiatry, 25(11), 2970-2978. https://doi.org/10.1038/s41380-019-0454-9

Baron-Cohen, S., \& Wheelwright, S. (2004). The empathy quotient: An investigation of adults with Asperger syndrome or high functioning autism, and normal sex differences. Journal of Autism and Developmental Disorders, 34(2), 163-175. https://doi.org/10.1023/b:jadd.0000022607.19833.00

Baron-Cohen, S., Wheelwright, S., Skinner, R., Martin, J., \& Clubley, E. (2001). The AutismSpectrum Quotient (AQ): Evidence from Asperger syndrome/high-functioning autism, males and females, scientists and mathematicians. Journal of Autism and Developmental Disorders, 31(1), 5-17. https://doi.org/10.1023/A:1005653411471

Bejerot, S., \& Eriksson, J. M. (2014). Sexuality and gender role in autism spectrum disorder: A case control study. PLoS ONE, 9(1), e87961. https://doi.org/10.1371/journal.pone.0087961

Brugha, T. S., Spiers, N., Bankart, J., Cooper, S.-A., McManus, S., Scott, F. J., Smith, J., \& Tyrer, F. (2016). Epidemiology of autism in adults across age groups and ability levels. British Journal of Psychiatry, 209(6), 498-503. https://doi.org/10.1192/bjp.bp.115.174649 
Chapman, E., Baron-Cohen, S., Auyeung, B., Knickmeyer, R. C., Taylor, K., \& Hackett, G. (2006). Fetal testosterone and empathy: Evidence from the empathy quotient (EQ) and the "reading the mind in the eyes" test. Social Neuroscience, 1(2), 135-148. https://doi.org/10.1080/17470910600992239

Cohen, J. (1988). Statistical power analysis for the behavioral sciences (2nd ed.). Lawrence Erlbaum Associates.

Coleman-Smith, R. S., Smith, R., Milne, E., \& Thompson, A. R. (2020). 'Conflict versus congruence': A qualitative study exploring the experience of gender dysphoria for adults with autism spectrum disorder. Journal of Autism and Developmental Disorders, 50(8), 2643-2657. https://doi.org/10.1007/s10803-019-04296-3

Cooper, K., Smith, L. G. E., \& Russell, A. J. (2018). Gender identity in autism: Sex differences in social affiliation with gender groups. Journal of Autism and Developmental Disorders, 48(12), 3995-4006. https://doi.org/10.1007/s10803-018-3590-1

de Vries, A. L. C., Noens, I. L. J., Cohen-Kettenis, P. T., van Berckelaer-Onnes, I. A., \& Doreleijers, T. A. (2010). Autism spectrum disorders in gender dysphoric children and adolescents. Journal of Autism and Developmental Disorders, 40(8), 930-936. https://doi.org/10.1007/s10803-010-0935-9

Dewinter, J., De Graaf, H., \& Begeer, S. (2017). Sexual orientation, gender identity, and romantic relationships in adolescents and adults with autism spectrum disorder. Journal of Autism and Developmental Disorders, 47(9), 2927-2934. https://doi.org/10.1007/s10803-017-3199-9

Di Ceglie, D., Skagerberg, E., Baron-Cohen, S., \& Auyeung, B. (2014). Empathising and systemising in adolescents with gender dysphoria. Opticon1826, 16(6), 1-8. https://doi.org/10.5334/opt.bo 
Dworzynski, K., Ronald, A., Bolton, P., \& Happé, F. (2012). How different are girls and boys above and beloe the diagnostic threshold for autism spectrum disorders? Journal of the American Academy of Child \& Adolescent Psychiatry, 51(8), 788-797. https://doi.org/10.1016/j.jaac.2012.05.018

Fombonne, E. (2009). Epidemiology of pervasive developmental disorders. Pediatric Research, 65(6), 591-598. https://doi.org/10.1203/pdr.0b013e31819e7203

George, R., \& Stokes, M. A. (2018). Gender identity and sexual orientation in autism spectrum disorder. Autism, 22(8), 970-982. https://doi.org/10.1177/1362361317714587

Glidden, D., Bouman, W. P., Jones, B. A., \& Arcelus, J. (2016). Gender dysphoria and autism spectrum disorder: A systematic review of the literature. Sexual Medicine Reviews, 4(1), 3-14. https://doi.org/10.1016/j.sxmr.2015.10.003

Greenberg, D. M., Warrier, V., Allison, C., \& Baron-Cohen, S. (2018). Testing the Empathizing-Systemizing theory of sex differences and the Extreme Male Brain theory of autism in half a million people. Proceedings of the National Academy of Sciences of the United States of America, 115(48), 12152-12157. https://doi.org/10.1073/pnas.1811032115

Griffiths, S., Allison, C., Kenny, R., Holt, R., Smith, P., \& Baron-Cohen, S. (2019). The Vulnerability Experiences Quotient (VEQ): A study of vulnerability, mental health and life satisfaction in autistic adults. Autism Research, 12(10), 1516-1528. https://doi.org/10.1002/aur.2162

Heylens, G., Aspeslagh, L., Dierickx, J., Baetens, K., Van Hoorde, B., De Cuypere, G., \& Elaut, E. (2018). The co-occurrence of gender dysphoria and autism spectrum disorder in adults: An analysis of cross-sectional and clinical chart data. Journal of Autism and Developmental Disorders, 48(6), 2217-2223. https://doi.org/10.1007/s10803-018-3480- 
Hines, M. (2004). Brain gender. Oxford University Press.

Hisle-Gorman, E., Landis, C. A., Susi, A., Schvey, N. A., Gorman, G. H., Nylund, C. M., \& Klein, D. A. (2019). Gender dysphoria in children with autism spectrum disorder. LGBT Health, 6(3), 95-100. https://doi.org/10.1089/lgbt.2018.0252

Hoekstra, R. A., Bartels, M., Cath, D. C., \& Boomsma, D. I. (2008). Factor structure, reliability and criterion validity of the autism-spectrum quotient (AQ): A study in Dutch population and patient groups. Journal of Autism and Developmental Disorders, 38(8), 1555-1566. https://doi.org/10.1007/s10803-008-0538-x

Hollocks, M. J., Lerh, J. W., Magiati, I., Meiser-Stedman, R., \& Brugha, T. S. (2019). Anxiety and depression in adults with autism spectrum disorder: A systematic review and metaanalysis. $\quad$ Psychological $\quad$ Medicine, $\quad 49(4), \quad$ 559-572. https://doi.org/10.1017/s0033291718002283

Hull, L., Petrides, K. V., \& Mandy, W. (2020). The female autism phenotype and camouflaging: A narrative review. Review Journal of Autism and Developmental Disorders. https://doi.org/10.1007/s40489-020-00197-9

Ingudomnukul, E., Baron-Cohen, S., Wheelwright, S., \& Knickmeyer, R. C. (2007). Elevated rates of testosterone-related disorders in women with autism spectrum conditions. Hormones and Behavior, 51(5), 597-604. https://doi.org/10.1016/j.yhbeh.2007.02.001

Jacobs, L. A., Rachlin, K., Erickson-Schroth, L., \& Janssen, A. (2014). Gender dysphoria and co-occurring autism spectrum disorders: Review, case examples, and treatment considerations. LGBT Health, 1(4), 277-282. https://doi.org/10.1089/lgbt.2013.0045

Jones, R. M., Wheelwright, S., Farrell, K., Martin, E., Green, R., Di Ceglie, D., \& BaronCohen, S. (2012). Brief report: Female-to-male transsexual people and autistic traits. 
Journal of Autism and Developmental Disorders, 42(2), 301-306. https://doi.org/10.1007/s10803-011-1227-8

Kanfiszer, L., Davies, F., \& Collins, S. (2017). 'I was just so different': The experiences of women diagnosed with an autism spectrum disorder in adulthood in relation to gender and social relationships. Autism, 21(6), 661-669. https://doi.org/10.1177/1362361316687987

Kristensen, Z. E., \& Broome, M. R. (2015). Autistic traits in an Internet sample of gender variant UK adults. International Journal of Transgenderism, 16(4), 234-245. https://doi.org/10.1080/15532739.2015.1094436

Kung, K. T. F. (2020). Autistic traits, systemising, empathising, and theory of mind in transgender and non-binary adults. Molecular Autism, 11(1), 73. https://doi.org/10.1186/s13229-020-00378-7

Kung, K. T. F., Spencer, D., Pasterski, V. L., Neufeld, S. A. S., Glover, V., Connor, T. G. O., Hindmarsh, P. C., Hughes, I. A., Acerini, C. L., \& Hines, M. (2016). No relationship between prenatal androgen exposure and autistic traits: Convergent evidence from studies of children with congenital adrenal hyperplasia and of amniotic testosterone concentrations in typically developing children. Journal of Child Psychology and Psychiatry, 57(12), 1455-1462. https://doi.org/10.1111/jcpp.12602

Lai, M.-C., Lombardo, M. V., Ruigrok, A. N., Chakrabarti, B., Auyeung, B., Szatmari, P., Happé, F., \& Baron-Cohen, S. (2017). Quantifying and exploring camouflaging in men and women with autism. Autism, 21(6), 690-702. https://doi.org/10.1177/1362361316671012

Landén, M., \& Rasmussen, P. (1997). Gender identity disorder in a girl with autism - a case report. European Child \& Adolescent Psychiatry, 6(3), 170-173. https://doi.org/10.1007/s007870050026 
Leef, J. H., Brian, J., VanderLaan, D. P., Wood, H., Scott, K., Lai, M.-C., Bradley, S. J., \& Zucker, K. J. (2019). Traits of autism spectrum disorder in school-aged children with gender dysphoria: A comparison to clinical controls. Clinical Practice in Pediatric Psychology, 7(4), 383-395. https://doi.org/10.1037/cpp0000303

Loomes, R., Hull, L., \& Mandy, W. P. L. (2017). What is the male-to-female ratio in autism spectrum disorder? A systematic review and meta-analysis. Journal of the American Academy of Child \& Adolescent Psychiatry, 56(6), 466-474. https://doi.org/10.1016/j.jaac.2017.03.013

Manning, J. T., Baron-Cohen, S., Wheelwright, S., \& Fink, B. (2010). Is digit ratio (2D:4D) related to systemizing and empathizing? Evidence from direct finger measurements reported in the BBC internet survey. Personality and Individual Differences, 48(6), 767771. https://doi.org/10.1016/j.paid.2010.01.030

Manning, J. T., Reimers, S., Baron-Cohen, S., Wheelwright, S., \& Fink, B. (2010). Sexually dimorphic traits (digit ratio, body height, systemizing-empathizing scores) and gender segregation between occupations: Evidence from the BBC internet study. Personality and Individual Differences, 49(5), 511-515. https://doi.org/10.1016/j.paid.2010.05.015

Manning, J. T., Trivers, R., \& Fink, B. (2020). Digit ratio (2D:4D), transgendered belief, and transsexual drug therapy in the BBC Internet Study. Evolutionary Psychological Science, 6(4), 380-388. https://doi.org/10.1007/s40806-020-00247-9

Meyer-Bahlburg, H. F. L. (2005). Gender identity outcome in female-raised 46,XY persons with penile agenesis, cloacal exstrophy of the bladder, or penile ablation. Archives of Sexual Behavior, 34(4), 423-438. https://doi.org/10.1007/s10508-005-4342-9

Millet, N., Longworth, J., \& Arcelus, J. (2017). Prevalence of anxiety symptoms and disorders in the transgender population: A systematic review of the literature. International Journal 
of Transgenderism, 18(1), 27-38. https://doi.org/10.1080/15532739.2016.1258353

Nieder, T. O., Herff, M., Cerwenka, S., Preuss, W. F., Cohen-Kettenis, P. T., De Cuypere, G., Hebold Haraldsen, I. R., \& Richter-Appelt, H. (2011). Age of onset and sexual orientation in transsexual males and females. Journal of Sexual Medicine, 8(3), 783-791. https://doi.org/10.1111/j.1743-6109.2010.02142.x

Nobili, A., Glazebrook, C., Bouman, W. P., Baron-Cohen, S., \& Arcelus, J. (2020). The stability of autistic traits in transgender adults following cross-sex hormone treatment. International Journal of Transgender Health, 21(4), 431-439. https://doi.org/10.1080/26895269.2020.1783738

Nobili, A., Glazebrook, C., Bouman, W. P., Glidden, D., Baron-Cohen, S., Allison, C., Smith, P., \& Arcelus, J. (2018). Autistic traits in treatment-seeking transgender adults. Journal of Autism and Developmental Disorders, 48(12), 3984-3994. https://doi.org/10.1007/s10803-018-3557-2

Øien, R. A., Cicchetti, D. V., \& Nordahl-Hansen, A. (2018). Gender dysphoria, sexuality and autism spectrum disorders: A systematic map review. Journal of Autism and Developmental Disorders, 48(12), 4028-4037. https://doi.org/10.1007/s10803-018-36867

Pasterski, V. L., Gilligan, L., \& Curtis, R. (2014). Traits of autism spectrum disorders in adults with gender dysphoria. Archives of Sexual Behavior, 43(2), 387-393. https://doi.org/10.1007/s10508-013-0154-5

Ratto, A. B. (2021). Commentary: What's so special about girls on the autism spectrum? - a commentary on Kaat et al. (2020). Journal of Child Psychology and Psychiatry, 62(1), 107-109. https://doi.org/10.1111/jcpp.13284

Reimers, S. (2007). The BBC Internet Study: General methodology. Archives of Sexual 
Behavior, 36(2), 147-161. https://doi.org/10.1007/s10508-006-9143-2

Richards, G., Wei, Y., \& Hendriks, O. (2020). Self-measure digit ratio (2D:4D) and gender variance. Endocrine Practice, 26(2), 250-251. https://doi.org/10.4158/1934-240326.2.250

Ruzich, E., Allison, C., Smith, P., Watson, P., Auyeung, B., Ring, H., \& Baron-Cohen, S. (2015). Measuring autistic traits in the general population: A systematic review of the Autism-Spectrum Quotient (AQ) in a nonclinical population sample of 6,900 typical adult males and females. Molecular Autism, 6(1), 2. https://doi.org/10.1186/2040-2392-6-2

Sala, G., Pecora, L., Hooley, M., \& Stokes, M. A. (2020). As diverse as the spectrum itself: Trends in sexuality, gender and autism. Current Developmental Disorders Reports, 7(2), 59-68. https://doi.org/10.1007/s40474-020-00190-1

Skagerberg, E., Di Ceglie, D., \& Carmichael, P. (2015). Brief report: Autistic features in children and adolescents with gender dysphoria. Journal of Autism and Developmental Disorders, 45(8), 2628-2632. https://doi.org/10.1007/s10803-015-2413-x

Stagg, S. D., \& Vincent, J. (2019). Autistic traits in individuals self-defining as transgender or nonbinary. European Psychiatry, 61, 17-22. https://doi.org/10.1016/j.eurpsy.2019.06.003

Strang, J. F., Kenworthy, L., Dominska, A., Sokoloff, J., Kenealy, L. E., Berl, M., Walsh, K., Menvielle, E., Slesaransky-Poe, G., Kim, K.-E., Luong-Tran, C., Meagher, H., \& Wallace, G. L. (2014). Increased gender variance in autism spectrum disorders and attention deficit hyperactivity disorder. Archives of Sexual Behavior, 43(8), 1525-1533. https://doi.org/10.1007/s10508-014-0285-3

Strang, J. F., Meagher, H., Kenworthy, L., de Vries, A. L. C., Menvielle, E., Leibowitz, S., Janssen, A., Cohen-Kettenis, P., Shumer, D. E., Edwards-Leeper, L., Pleak, R. R., Spack, N., Karasic, D. H., Schreier, H., Balleur, A., Tishelman, A., Ehrensaft, D., Rodnan, L., 
Kuschner, E. S., ... Anthony, L. G. (2018a). Initial clinical guidelines for co-occurring autism spectrum disorder and gender dysphoria or incongruence in adolescents. Journal of Clinical Child \& Adolescent Psychology, 47(1), 105-115. https://doi.org/10.1080/15374416.2016.1228462

Strang, J. F., Powers, M. D., Knauss, M., Sibarium, E., Leibowitz, S. F., Kenworthy, L., Sadikova, E., Wyss, S., Willing, L., Caplan, R., Pervez, N., Nowak, J., Gohari, D., GomezLobo, V., Call, D., \& Anthony, L. G. (2018b). "They thought It was an obsession": Trajectories and perspectives of autistic transgender and gender-diverse adolescents. Journal of Autism and Developmental Disorders, 48(12), 4039-4055. https://doi.org/10.1007/s10803-018-3723-6

Strang, J. F., van der Miesen, A. I. R., Caplan, R., Hughes, C., DaVanport, S., \& Lai, M.-C. (2020). Both sex- and gender-related factors should be considered in autism research and clinical practice. Autism, 24(3), 539-543. https://doi.org/10.1177/1362361320913192

Valentine, S. E., \& Shipherd, J. C. (2018). A systematic review of social stress and mental health among transgender and gender non-conforming people in the United States. Clinical Psychology Review, 66, 24-38. https://doi.org/10.1016/j.cpr.2018.03.003

van der Miesen, A. I. R., Hurley, H., Bal, A. M., \& de Vries, A. L. C. (2018). Prevalence of the wish to be of the opposite gender in adolescents and adults with autism spectrum disorder. Archives of Sexual Behavior, 47(8), 2307-2317. https://doi.org/10.1007/s10508018-1218-3

van der Miesen, A. I. R., Hurley, H., \& de Vries, A. L. C. (2016). Gender dysphoria and autism spectrum disorder: A narrative review. International Review of Psychiatry, 28(1), 70-80. https://doi.org/10.3109/09540261.2015.1111199

Vermaat, L. E. W., van der Miesen, A. I. R., de Vries, A. L. C., Steensma, T. D., Popma, A., 
Cohen-Kettenis, P. T., \& Kreukels, B. P. C. (2018). Self-reported autism spectrum disorder symptoms among adults referred to a gender identity clinic. LGBT Health, 5(4), 226-233. https://doi.org/10.1089/lgbt.2017.0178

Wakabayashi, A., Baron-Cohen, S., Wheelwright, S., Goldenfeld, N., Delaney, J., Fine, D., Smith, R., \& Weil, L. (2006). Development of short forms of the Empathy Quotient (EQShort) and the Systemizing Quotient (SQ-Short). Personality and Individual Differences, 41(5), 929-940. https://doi.org/10.1016/j.paid.2006.03.017

Warrier, V., Greenberg, D. M., Weir, E., Buckingham, C., Smith, P., Lai, M. C., Allison, C., \& Baron-Cohen, S. (2020). Elevated rates of autism, other neurodevelopmental and psychiatric diagnoses, and autistic traits in transgender and gender-diverse individuals. Nature Communications, 11(1), 1-12. https://doi.org/10.1038/s41467-020-17794-1

Woodbury-Smith, M. R., Robinson, J., Wheelwright, S., \& Baron-Cohen, S. (2005). Screening adults for Asperger Syndrome using the AQ: A preliminary study of its diagnostic validity in clinical practice. Journal of Autism and Developmental Disorders, 35(3), 331-335. https://doi.org/10.1007/s10803-005-3300-7

Zhang, Q., Goodman, M., Adams, N., Corneil, T., Hashemi, L., Kreukels, B., Motmans, J., Snyder, R., \& Coleman, E. (2020). Epidemiological considerations in transgender health: A systematic review with focus on higher quality data. International Journal of Transgender Health, 21(2), 125-137. https://doi.org/10.1080/26895269.2020.1753136

Zucker, K. J. (2017). Epidemiology of gender dysphoria and transgender identity. Sexual Health, 14(5), 404-411. https://doi.org/10.1071/SH17067

Zucker, K. J., \& Lawrence, A. A. (2009). Epidemiology of gender identity disorder: Recommendations for the standards of care of the world professional association for transgender health. International Journal of Transgenderism, 11(1), 8-18. 
https://doi.org/10.1080/15532730902799946

Zucker, K. J., Nabbijohn, A. N., Santarossa, A., Wood, H., Bradley, S. J., Matthews, J., \& VanderLaan, D. P. (2017). Intense/obsessional interests in children with gender dysphoria: A cross-validation study using the Teacher's Report Form. Child and Adolescent Psychiatry and Mental Health, 11(1). https://doi.org/10.1186/s13034-0170189-9 
Table 1. Demographic information for the whole sample and stratified by gender group.

\begin{tabular}{|c|c|c|c|c|c|c|c|c|c|c|c|c|c|}
\hline & & \multicolumn{3}{|c|}{ Transgender AFAB } & \multicolumn{3}{|c|}{ Cisgender Female } & \multicolumn{3}{|c|}{ Transgender AMAB } & \multicolumn{3}{|c|}{ Cisgender Male } \\
\hline & & $N$ & $M$ & $S D$ & $N$ & $M$ & $S D$ & $N$ & $M$ & $S D$ & $N$ & $M$ & $S D$ \\
\hline \multirow[t]{2}{*}{ Age } & & 32 & 30.34 & 11.24 & 21 & 33.57 & 12.91 & 18 & 43.50 & 15.49 & 18 & 40.72 & 17.92 \\
\hline & & $N$ & $\%$ & & $N$ & $\%$ & & $N$ & $\%$ & & $N$ & $\%$ & \\
\hline \multirow[t]{4}{*}{ Sexual Orientation } & Heterosexual & 0 & $0.0 \%$ & & 11 & $52.4 \%$ & & 4 & $22.2 \%$ & & 9 & $50.0 \%$ & \\
\hline & Homosexual & 4 & $12.5 \%$ & & 6 & $28.6 \%$ & & 4 & $22.2 \%$ & & 6 & $33.3 \%$ & \\
\hline & Bisexual & 14 & $43.8 \%$ & & 3 & $14.3 \%$ & & 4 & $22.2 \%$ & & 2 & $11.1 \%$ & \\
\hline & Other/Prefer not to say & 14 & $43.8 \%$ & & 1 & $4.8 \%$ & & 6 & $33.3 \%$ & & 1 & $5.6 \%$ & \\
\hline \multirow[t]{2}{*}{ Ethnicity } & White & 26 & $81.3 \%$ & & 16 & $76.2 \%$ & & 16 & $88.9 \%$ & & 16 & $88.9 \%$ & \\
\hline & Not White & 6 & $18.8 \%$ & & 5 & $23.8 \%$ & & 2 & $11.1 \%$ & & 2 & $11.1 \%$ & \\
\hline \multirow[t]{4}{*}{ Autism $^{1}$} & Not diagnosed or suspected & 18 & $56.3 \%$ & & 15 & $71.4 \%$ & & 14 & $77.8 \%$ & & 17 & $94.4 \%$ & \\
\hline & Diagnosed & 6 & $18.8 \%$ & & 4 & $19.0 \%$ & & 1 & $5.6 \%$ & & 1 & $5.6 \%$ & \\
\hline & Suspected & 9 & $28.1 \%$ & & 5 & $23.8 \%$ & & 3 & $16.7 \%$ & & 1 & $5.6 \%$ & \\
\hline & Diagnosed and/or suspected & 14 & $43.8 \%$ & & 6 & $28.6 \%$ & & 4 & $22.2 \%$ & & 1 & $5.6 \%$ & \\
\hline \multirow[t]{3}{*}{ Gender Dysphoria $^{2}$} & Not diagnosed or suspected & 5 & $15.6 \%$ & & 21 & $100.0 \%$ & & 2 & $11.1 \%$ & & 18 & $100.0 \%$ & \\
\hline & Diagnosed & 17 & $53.1 \%$ & & 0 & $0.0 \%$ & & 11 & $61.1 \%$ & & 0 & $0.0 \%$ & \\
\hline & Suspected & 16 & $50.0 \%$ & & 0 & $0.0 \%$ & & 9 & $50.0 \%$ & & 0 & $0.0 \%$ & \\
\hline
\end{tabular}


Diagnosed and/or suspected

$2784.4 \%$

$0 \quad 0.0 \%$

$16 \quad 88.9 \%$

$0 \quad 0.0 \%$

Recruited via LGBT group? Yes

$17 \quad 54.8 \%$

1

$5.0 \%$

10

$55.6 \%$

6

$33.3 \%$

No

$14 \quad 45.2 \%$

$19 \quad 95.0 \%$

$844.4 \%$

$1266.7 \%$

Note. Transgender AFAB = assigned female at birth but does not identify as female; Transgender AMAB = assigned male at birth but does not identify as male.

${ }^{1}$ Some participants specified both diagnosed and suspected options for autism; the "diagnosed and/or suspected" category includes all participants who responded in the affirmative to either or both questions.

${ }^{2}$ Some participants specified both diagnosed and suspected options for gender dysphoria; the "diagnosed and/or suspected" category includes all participants who responded in the affirmative to either or both questions. 
Table 2. Autistic traits, empathizing, systemizing, and D-scores across gender groups.

\begin{tabular}{lllllllllllll} 
& AQ & \multicolumn{3}{c}{ EQ-S } & \multicolumn{3}{c}{ SQ-S } & \multicolumn{3}{c}{ D-score } \\
& $n$ & $M$ & $S D$ & $n$ & $M$ & $S D$ & $n$ & $M$ & $S D$ & $n$ & $M$ & $S D$ \\
& & & & & & & & & & & & \\
& 21 & 19.43 & 9.93 & 19 & 21.05 & 10.82 & 19 & 13.74 & 9.68 & 19 & -0.07 & 0.16 \\
\hline Cisgender female & & & & & & & & & & & & \\
Transgender AFAB & 32 & 25.88 & 10.25 & 30 & 16.87 & 10.03 & 29 & 22.66 & 9.28 & 29 & 0.06 & 0.16 \\
\hline
\end{tabular}

$\begin{array}{lcccccccccccc}\text { Cisgender male } & 18 & 18.11 & 7.61 & 18 & 20.83 & 10.00 & 18 & 18.94 & 6.82 & 18 & -0.02 & 0.15 \\ \text { Transgender AMAB } & 18 & 20.17 & 9.06 & 18 & 22.06 & 9.01 & 17 & 21.24 & 9.54 & 17 & -0.01 & 0.16\end{array}$

Note. Transgender AFAB = assigned female at birth but does not identify as female; Transgender AMAB = assigned male at birth but does not identify as male; $\mathrm{AQ}=\mathrm{Autism}$ Spectrum Quotient total score (scores can range from 0 to 50); EQ-S = Empathy Quotient-Short total score (scores can range from = 0 to 44 ); SQ-S = Systemizing QuotientShort total score (scores can range from 0 to 50 ); D-score = difference between standardized EQ-S (E) and standardized SQ-S (S) scores. 
Table 3. Outcome of ANCOVA models with assigned gender, current gender, and the assigned $\times$ current gender interaction term as predictors, autism status and age as covariates, and AQ, EQ-S, SQ-S, and D-score as outcomes.

\begin{tabular}{llllllllllllll} 
& AQ & \multicolumn{4}{c}{ EQ-S } & \multicolumn{3}{c}{ SQ-S } & \multicolumn{5}{c}{ D-score } \\
\hline & $F$ & $p$ & $\eta_{\mathrm{p}}{ }^{2}$ & $F$ & $p$ & $\eta_{\mathrm{p}}{ }^{2}$ & $F$ & $p$ & $\eta_{\mathrm{p}}{ }^{2}$ & $F$ & $p$ & $\eta_{\mathrm{p}}{ }^{2}$ \\
\hline Assigned gender & 0.65 & 0.42 & 0.01 & $<0.01$ & 0.98 & $<0.001$ & 0.83 & 0.37 & 0.01 & 0.30 & 0.59 & $<0.01$ \\
Current gender & 1.57 & 0.21 & 0.02 & 0.06 & 0.81 & $<0.01$ & 5.56 & 0.02 & 0.07 & 1.32 & 0.26 & 0.02 \\
Assigned $\times$ current gender & 2.28 & 0.14 & 0.03 & 2.23 & 0.14 & 0.03 & 3.05 & 0.09 & 0.04 & 4.78 & 0.03 & 0.06 \\
Age & 2.07 & 0.15 & 0.02 & 0.43 & 0.51 & 0.01 & 1.38 & 0.24 & 0.02 & 1.70 & 0.20 & 0.02 \\
Autism status & 43.38 & $<0.001$ & 0.34 & 27.20 & $<0.001$ & 0.26 & 2.64 & 0.11 & 0.03 & 22.51 & $<0.001$ & 0.23 \\
\hline
\end{tabular}

Note. Degrees of freedom for each ANCOVA model are as follow: AQ $(1,83)$; EQ-S $(1,79)$; SQ-S $(1,77)$; D-score $(1,77)$. 
Figure 1. Interaction between gender assigned at birth and current gender on the standardized mean difference between empathizing and systemizing (D-score)

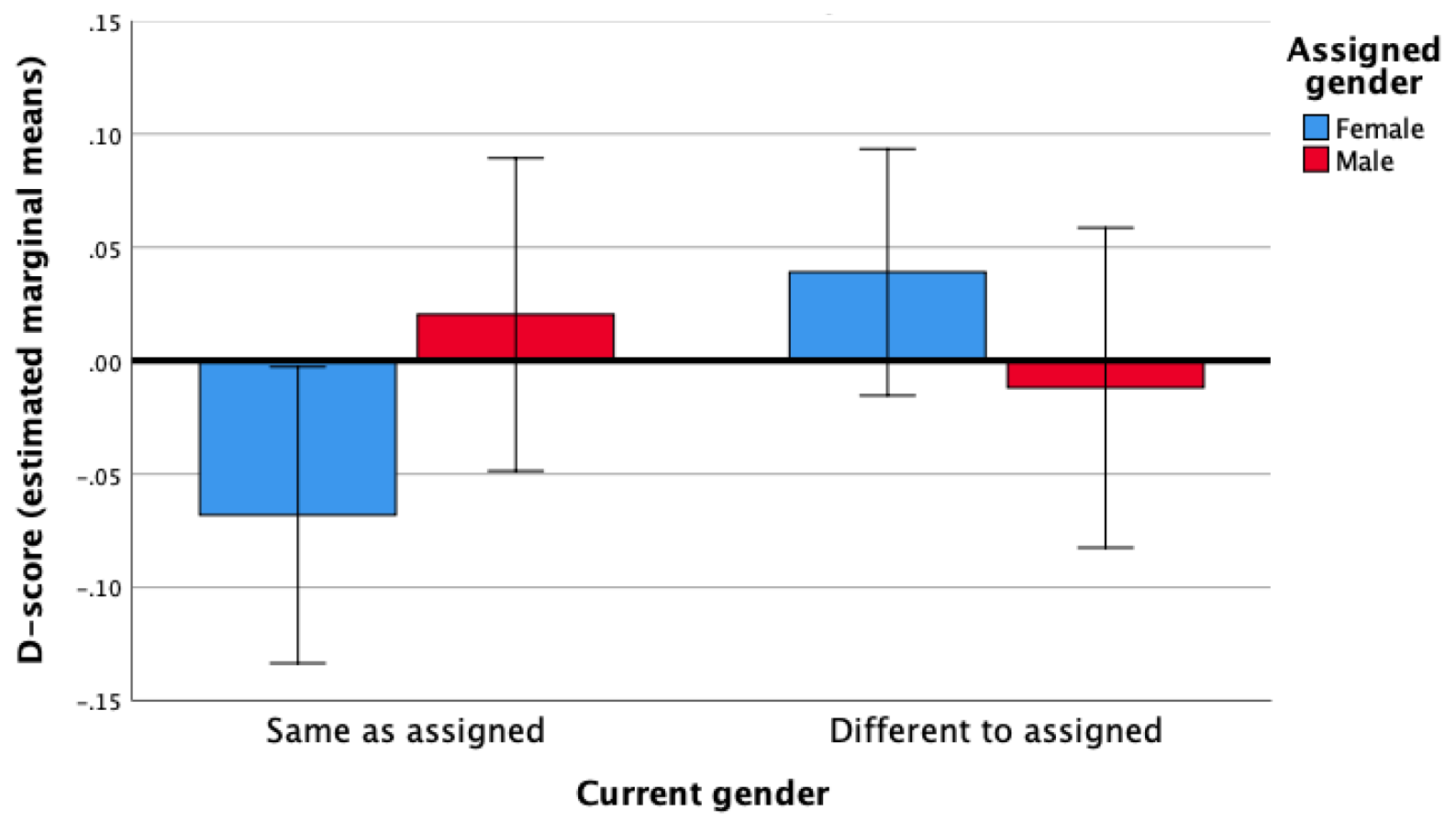

Note. High D-scores indicate high systemizing (SQ-S total score) relative to empathizing (EQ-S total score); age and diagnosed/suspected autism were controlled for as covariates; error bars are $95 \% \mathrm{CI}$. 